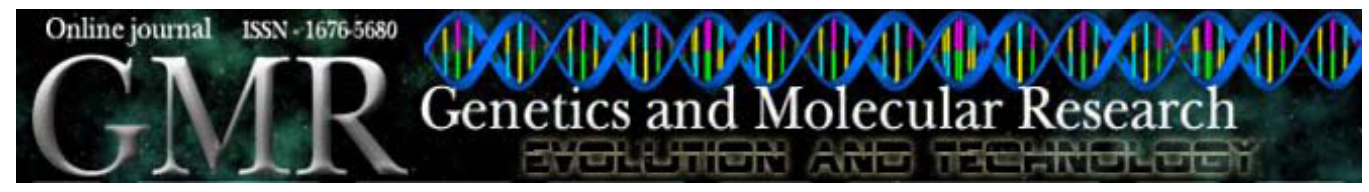

\title{
Detection of deletions and duplications in the Duchenne muscular dystrophy gene by the molecular method MLPA in the first Argentine affected families
}

\author{
D.M. Marzese' ${ }^{1}$, A. Mampel ${ }^{2}$, L.C. Gomez ${ }^{1}$, M.I. Echeverria ${ }^{2}$, \\ A.L. Vargas $^{2}$, V. Ferreiro ${ }^{3}$, F. Giliberto ${ }^{3}$ and M. Roqué ${ }^{1}$ \\ ${ }^{1}$ Laboratory of Cellular and Molecular Biology, IHEM-CONICET, \\ School of Medical Sciences, National University of Cuyo, Mendoza, Argentina \\ ${ }^{2}$ Genetics Institute, School of Medical Sciences, \\ National University of Cuyo, Mendoza, Argentina \\ ${ }^{3}$ Department of Molecular Genetics, Clinical Hospital, \\ University of Buenos Aires, Argentina \\ Corresponding author: M. Roqué \\ E-mail: mroque@fcm.uncu.edu.ar
}

Genet. Mol. Res. 7 (1): 223-233 (2008)

Received October 24, 2007

Accepted December 28, 2007

Published March 11, 2008

\begin{abstract}
Deletions/duplications in the Duchenne muscular dystrophy (DMD) gene account for 60 to $70 \%$ of all alterations. A new technique, multiplex ligation-dependent probe amplification (MLPA), has been described that allows the detection of large genetic rearrangements by simultaneous amplification of up to 45 target sequences. The present article is based on the diagnosis of the first Argentine affected families by the application of MLPA. DNA samples from patients with and without a previous diagnosis were included. MLPA assays were performed according to manufacturer recommendations. Polymerase chain reaction and direct sequencing were performed when a single-exon deletion was detected. Results
\end{abstract}


were analyzed using the Gene Marker v1.6 and Sequencing Analysis v5.2 software. In the samples with a previous diagnosis (as identified by short tandem repeat-polymerase chain reaction analysis), MLPA confirmed in some samples the same deletion and detected in others a larger deleted fragment. This enabled the prediction of the expected male phenotype. One deletion and one duplication were detected in patients without previous diagnosis. In this study, we investigated the applicability of MLPA in our country. Our results showed a $100 \%$ confirmation of the deleted fragments detected by short tandem repeat segregation analysis. Moreover, in some cases, the MLPA assay was able to refine the breakpoints involved. In addition, MLPA identified deletions/duplications in samples without previous diagnosis. In comparison to the available diagnosis strategies in Argentina, MLPA is less time-consuming, and spans the complete coding region of DMD. The application of MLPA will improve the genetic diagnosis of DMD/Becker muscular dystrophy in our country.

Key words: Duchenne muscular dystrophy; Becker muscular dystrophy; Deletions/duplications; Multiplex ligation-dependent probe amplification

\section{INTRODUCTION}

Duchenne muscular dystrophy (DMD) and Becker muscular dystrophy (BMD) are the second most commonly occurring genetically inherited diseases. They are X-linked disorders related to mutations in the DMD gene, with Duchenne patients presenting a more severe phenotype than Becker patients. The related DMD gene, located at Xp21, is the largest identified gene in the human genome, spanning $2.4 \mathrm{Mb}$ and 79 exons. It has a high mutation rate, with approximately one-third of all cases resulting from a spontaneous mutation. Its transcript is $14 \mathrm{~kb}$ long and is expressed in skeletal muscle and brain (Hoffman et al., 1987; Den Dunnen et al., 1992). The protein product of the DMD gene, called dystrophin, is involved in the linkage between the extracellular matrix and the cellular cytoskeleton (Yoshida and Ozawa, 1990; Ervasti and Campbell, 1991, 1993). The absence of dystrophin has been postulated to result in the lack of a mechanical link with the dystrophin-glycoprotein complex, and therefore results in contraction-induced muscle degeneration as a consequence of a decrease in muscle plasma membrane stability and disruptions in the sarcolemma (Hoffman et al., 1987; Petrof, 1998). Mutations that produce the complete absence of the protein, or that produce truncated dystrophins are related to the DMD phenotype, affecting mainly the respiratory and cardiac functions in boys in advanced stages of the disease. These mutations can affect the complete transcription of the gene or can produce truncated proteins which are unable to develop their cellular function. The incidence of DMD is around 1 in 3500 live-born males.

The dysfunction of dystrophin produces a milder form of the disease, called BMD, with a later age of onset and a slower clinical progression. The incidence of BMD is around 1:18,000 live-born males (Bushby et al., 1991). Mutations related to BMD affect the middle 
part of the gene, producing a shorter protein that conserves its amino- and carboxy-terminal domains to link the extracellular matrix with the intracellular cytoskeleton.

According to Western literature, partial deletions and duplications of the DMD gene account for 60 to 70\% of all DMD and BMD cases (Koenig et al., 1987; Den Dunnen et al., 1989). More than $98 \%$ of deletions of the DMD gene are readily detectable in affected males by using a multiplex polymerase chain reaction (PCR) approach based on an exon by exon analysis strategy within two hot-spot regions (exons 2-20 and 44-53) (Chamberlain et al., 1988; Den Dunnen et al., 1989; Beggs et al., 1990). The deletions are easy to visualize by PCR-based procedures in males, because of their hemizygous condition for this gene. The analysis is based on a discrimination between the presence or absence of a PCR product. The duplications of exons, however, cannot be visualized by PCR-based assays, due to the non-quantitative nature of the technique. Southern blotting has been the most conventional method used for the detection of such rearrangements; however, it is very labor-intensive and expensive.

Since no effective treatment is available for these diseases at present, the identification of carrier females is critical in order to provide appropriate genetic counseling. Indeed, in the families with a single affected male, a crucial point is to determine whether the disease is derived from a de novo mutation or from a genetic defect inherited from a carrier mother. The above described PCR approach is unable to detect either duplications or female carriers in whom deletions are masked by the amplification of the normal $\mathrm{X}$ chromosome. Thus, identification of female carriers requires different strategies, such as segregation analysis with intragenic polymorphic markers (Darras et al., 1987; Clemens et al., 1991), quantitative analysis of gene dosage (Prior et al., 1990; Abbs and Bobrow, 1993) and fluorescence in situ hybridization (FISH) analysis (Voskova-Goldman et al., 1997; Ligon et al., 2000). However, segregation analysis cannot be used in families with a single affected male or performed if DNA of the affected members of the family is not available. Quantitative analysis of gene dosage may be difficult to perform and interpret and, finally, FISH analysis can be unsuccessful when looking at partial gene deletions, and remains a low-throughput analysis compared with molecular genetic techniques. Hence, considerable effort has been made in recent years to develop new strategies for the rapid identification of female carriers of DMD deletions/duplications.

Recently, a new technique, multiplex ligation-dependent probe amplification (MLPA), has been described that allows the detection of large genetic rearrangements by simultaneous amplification of up to 45 target sequences (Schouten et al., 2002; Sellner and Taylor, 2004). In short, each MLPA probe consists of two oligonucleotides that hybridize to the target sequence at adjacent sites. These probes contain a short target-specific sequence (22-43 nucleotides) and a forward or reverse PCR primer-binding sequence that is universal to all the probes. In order to differentiate between different amplification products, the probes also contain a non-hybridizing stuffer sequence of variable length (19-364 nucleotides). After an overnight hybridization to the target DNA, consecutively hybridized probes are joined by a ligation reaction. The ligation products have both forward and reverse PCR primer-binding sites and can be amplified by PCR. The relative quantity of each of the PCR products is proportional to the number of copies of the target sequence. The different lengths of the products allow their separation on an automated capillary sequencer, where the peak areas are quantified (Schouten et al., 2002). 
This article is based on the application of the MLPA technique to detect exon deletions and duplications of the DMD gene. We assessed the efficacy of this technique by comparing the results with those from previously investigated patients who had been diagnosed using alternative methods. Our results indicate that MLPA is a powerful tool for the detection of female carriers and affected males.

\section{MATERIAL AND METHODS}

\section{Patients and female carriers}

This study included DNA from 9 female carriers and from 3 affected males. Six of the female carriers (DMD 1, 2, 3, 4, 8, 9) and one of the affected males (DMD 10) were identified with a previous short tandem repeat (STR) segregation analysis-based diagnosis (Table 1A). Three female carriers (DMD 5, 6, 7; Table 1B) and two affected males (DMD 11, 12; Table 1C) had not received a previous diagnosis. They were included in this study after signing a written informed consent. The female carrier DMD 7 is the mother of the affected male DMD 11, and the female carrier DMD 9 is the daughter of the affected male DMD 10.

\section{DNA samples}

The DNA samples were obtained by DNAzol ${ }^{\circledR}$ BD Reagent (Invitrogen) according to manufacturer recommendations and by the CTAB method. This last method consists, in brief, of the following steps: $3 \mathrm{~mL}$ whole blood was washed with $\mathrm{T}_{10} \mathrm{E}_{10}$ buffer and treated with CTAB solution (2\% CTAB, 0.2\% $\beta$-mercaptoethanol, $1.4 \mathrm{M} \mathrm{NaCl}, 20 \mathrm{mM}$ EDTA, $100 \mathrm{mM}$ Tris (buffer)). Subsequently, the DNA was purified with chloroform:isoamyl alcohol (24:1) and precipitated with $100 \%$ ethanol.

\section{Short tandem repeat analysis}

Eleven sets of primers were used to amplify the different intronic $\mathrm{CA}_{\mathrm{n}}$. All forward primers were labeled with $\gamma^{32}$ P-ATP. PCR reactions were carried out starting with 120 ng DNA. The products were resolved on $6 \%$ denaturing polyacrylamide gels and processed for autoradiography analysis. STR analysis was performed on the following female samples: DMD 1, 2, 3, 4, 8, and 9, and on the male sample DMD 10. These results have previously been presented for the defense of a $\mathrm{PhD}$ thesis (Ferreiro, 2006).

\section{Multiplex ligation-dependent probe amplification analysis}

MLPA analysis was performed on all the 12 samples included in this study. The MLPA assay for the DMD gene requires two different reactions due the large number of exons. One reaction contains a probe mix called PO34 that covers exons 1-10, 21-30, 41-50, and 61-70. The second reaction contains a probe mix called PO35 and covers the analysis of exons 11-20, 31-40, 51-60, and 71-79. The assay conditions were essentially according to manufacturer recommendations (MRC Holland, Amsterdam, The Netherlands). In order to set up the standard 
conditions in our laboratory, the starting amount of DNA was fixed at $250 \mathrm{ng}$. Briefly, $250 \mathrm{ng}$ DNA of each patient was denatured for $5 \mathrm{~min}$ at $98^{\circ} \mathrm{C}$ and hybridized overnight at $60^{\circ} \mathrm{C}$ with the SALSA probe mix PO34 and PO35. The hybridization products were subsequently treated with a ligase enzyme for $15 \mathrm{~min}$ at $54^{\circ} \mathrm{C}$. The ligation reaction was stopped by raising the temperature for $5 \mathrm{~min}$ at $98^{\circ} \mathrm{C}$. Finally, PCR amplification was carried out with the specific SALSA FAM primers using the ligation product as templates. PCR products were checked on a $2 \%$ agarose gel and subsequently resolved by capillary electrophoresis on an ABI PRISM 3130. The data were analyzed by the Gene Marker version 1.6 software.

\section{Polymerase chain reaction amplification and sequencing of exon 38}

The following primers were designed to amplify exon 38 of the DMD gene in sample DMD 11: 5' GGTTTATGTTTCTTATAAAAAGTAA and 5'CCACTCCTAGTTCATTCACAACCA. The PCR conditions consisted of 35 cycles with an annealing temperature of $50^{\circ} \mathrm{C}$ in $1.5 \mathrm{mM} \mathrm{MgCl}_{2}$ and $2.5 \mathrm{U}$ of GoTaq (Promega) in a final volume of $25 \mu \mathrm{L}$. The products were resolved on non-denaturing $10 \%$ polyacrylamide gel electrophoresis for $90 \mathrm{~min}$ at $120 \mathrm{~V}$, stained with ethidium bromide and exposed to UV light.

Direct sequencing was performed as follows: PCR products were purified from agarose gels with the DNA and Gel Band Purification Kit (GE Healthcare, UK). After the sequencing reaction with labeled ddNTPs, the products were resolved by capillary electrophoresis on the automated sequencer ABI3130. The results were analyzed using the Sequencing Analysis version 5.2 software.

\section{RESULTS}

In order to be able to set up the MLPA method and to compare the results obtained with previous diagnosis performed by STR analysis, DNA samples from six female carriers and one affected male were obtained from the Department of Molecular Genetics of the Clinical Hospital of the University of Buenos Aires. After obtaining informed consent, blood samples were taken from three female carriers and two affected males who had no previous molecular diagnosis by the Genetic Institute of the School of Medical Sciences of the National University of Cuyo. The MLPA, PCR, direct sequencing assays and software analyses of the results were performed at the Cellular and Molecular Laboratory of the School of Medical Sciences of the National University of Cuyo, Mendoza.

\section{MLPA confirms deletions detected by multiplex PCR and STR analysis}

This study included DNA samples from six female carriers, all with a previous STR-based diagnosis and one affected male with a direct analysis by multiplex PCR. The STR assays had been performed by the analysis of 11 (CA) intronic regions, located in the promoter region and in the introns $7 \mathrm{~A}, 25,44,44 \mathrm{~A}, 44 \mathrm{~B}, 45,49,50,63$, and 79 of the DMD gene. In the seven selected samples, the molecular analysis had identified different sizes of deletions, ranging in length from one to 18 deleted introns/exons. The MLPA analysis performed on these samples revealed the same deletion as detected by previous analysis (Table 1A) in three cases: DMD 1 (Figure 1A) and DMD 9 and 10 (electro- 
Table 1. Deletion/duplication detections by the multiplex ligation-dependent probe amplification (MLPA) assay.

\begin{tabular}{|c|c|c|c|c|c|c|c|}
\hline & Sample & Patient & $\begin{array}{l}\text { Deletion } \\
\text { detected } \\
\text { by STR }\end{array}$ & $\begin{array}{c}\text { Deletion } \\
\text { detected } \\
\text { by MLPA }\end{array}$ & $\begin{array}{l}\text { Refinement of } \\
\text { the involved } \\
\text { breakpoints }\end{array}$ & $\begin{array}{l}\text { Generation of } \\
\text { frameshift } \\
\text { according } \\
\text { to MLPA }\end{array}$ & $\begin{array}{c}\text { Expected phenotype } \\
\text { in males according } \\
\text { to MLPA }\end{array}$ \\
\hline \multirow[t]{7}{*}{ A. } & DMD 1 & Female carrier & Del exon 45-50 & Del exon 45-50 & No & Yes & DMD \\
\hline & DMD 2 & Female carrier & Del exon $18-25$ & Del exon $18-29$ & Yes & Yes & DMD \\
\hline & DMD 3 & Female carrier & Del exon 8-17 & Del exon 2-19 & Yes & No & BMD \\
\hline & DMD 4 & Female carrier & Del exon 8-17 & Del exon 2-18 & Yes & Yes & DMD \\
\hline & DMD 8 & Female carrier & Del intron 7 & Del exon 7-29 & Yes & Yes & DMD \\
\hline & DMD 9 & Female carrier & Del exon 45-55 & Del exon 45-55 & No & No & BMD \\
\hline & DMD 10 & Affected male & Del exon 45-55 & Del exon 45-55 & No & No & BMD \\
\hline \multirow[t]{3}{*}{ B. } & DMD 5 & Female carrier & - & Del exon 48-50 & - & Yes & DMD \\
\hline & DMD 6 & Female carrier & - & No del/dupl & - & - & - \\
\hline & DMD 7 & Female carrier & - & No del/dupl & - & - & - \\
\hline \multirow[t]{2}{*}{ C. } & DMD 11 & Affected male & - & $\begin{array}{l}\text { False del exon } \\
\text { 38/discarted by } \\
\text { PCR and } \\
\text { sequencing. }\end{array}$ & - & - & - \\
\hline & DMD 12 & Affected male & - & Dupl exon 47 & Yes & No & BMD \\
\hline
\end{tabular}

The included samples are clustered in three groups: A. Duchenne muscular dystrophy (DMD) 1, 2, 3, 4, 8, 9, and 10 are DNA samples with deletions previously detected by short tandem repeat (STR) analysis. Notice that in DMD 2, 3, 4 , and 8 , a larger deleted fragment is detected by MLPA. This result allowed analyzing the generation of a frame shift and consequently the expected phenotype in males with this mutation. B. DMD 5, 6 and 7 are female carriers without previous diagnosis. DMD 5's son had a polymerase chain reaction (PCR) diagnosis of a deletion detected only in exon 49. The MLPA analysis of the mother shows a wider deletion covering exons 48,49 and 50 . With this more precise delimitated mutation, it has been possible to infer the expected phenotype of the affected boy. DMD 7 is the mother of DMD 11. C. DMD 11 and 12 are affected males without previous diagnosis. DMD 11 showed a single exon (38) deletion by MLPA. In order to further analyze this apparent result, a subsequent PCR assay revealed no absence of this fragment. DMD 12 demonstrated by MLPA an exonic duplication, not detectable by PCR multiplex.
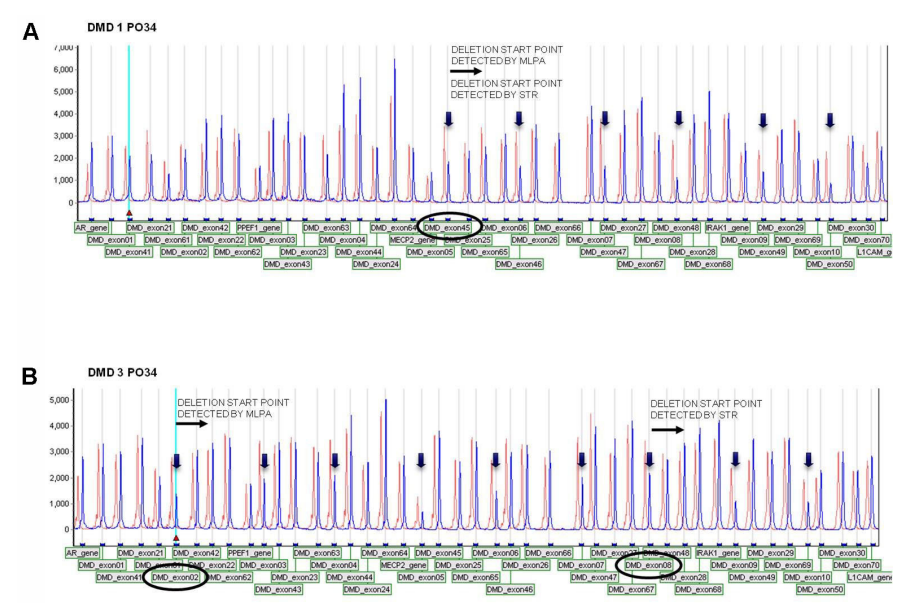

Figure 1. Multiplex ligation-dependent probe amplification (MLPA) analysis of two female carriers previously analyzed by a single-tandem repeat (STR) segregation. MLPA electropherograms of two female samples previously analyzed by STR (blue peaks) compared to a healthy female control (red peaks). The results were analyzed by the Gene Marker version 1.6 software. The height of each peak is correlated to the amount of DNA copies. Black arrows on the blue lower peaks indicate the half amount of copies for those sequences. A. DMD 1, the electropherogram shows a start point of the deletion on exon 45 , the same point as detected by the previous STR analysis. B. DMD 3 , the electropherogram shows the deletion detected by STR started on exon 8. MLPA allowed detecting a larger deleted fragment, starting on exon 2. 
pherograms not shown). These results confirmed that the MLPA assay was functioning properly and was set up correctly, and delimited the deleted exons to those detected by STR analysis.

\section{In some cases, MLPA reveals a larger fragment deleted in female carriers compared to detections based on STR analysis}

In the four remaining DNA samples which had been previously diagnosed by STR (DMD 2, DMD 3, DMD 4, and DMD 8), the MLPA analysis showed a larger deletion than that identified by STR segregation analysis (Table 1A and Figure 1B). Again, this confirmed the correct functioning of the method and showed an advantage: the delimitation of the deleted DNA fragment. This delimitation is essential for the phenotype prediction in affected males. When we analyzed the new delimitated mutation, we could predict an expected DMD or BMD phenotype in males, based on the analysis of the generation of frame shifts that could affect the open reading frame of the gene (Table 1A, B and C).

\section{MLPA allows the detection of deletions and duplications in samples without a previous diagnosis}

The detection by MLPA of deletions coincidently detected by STR segregation analysis encouraged us to subsequently apply this method on three new female patients (DMD 5, DMD 6, DMD 7) and on two affected males (DMD 11 and DMD 12) who could not have been diagnosed by STR analysis. A duplication of exon 47 was detected in one of the affected males (DMD 12, Table 1C and Figure 2).
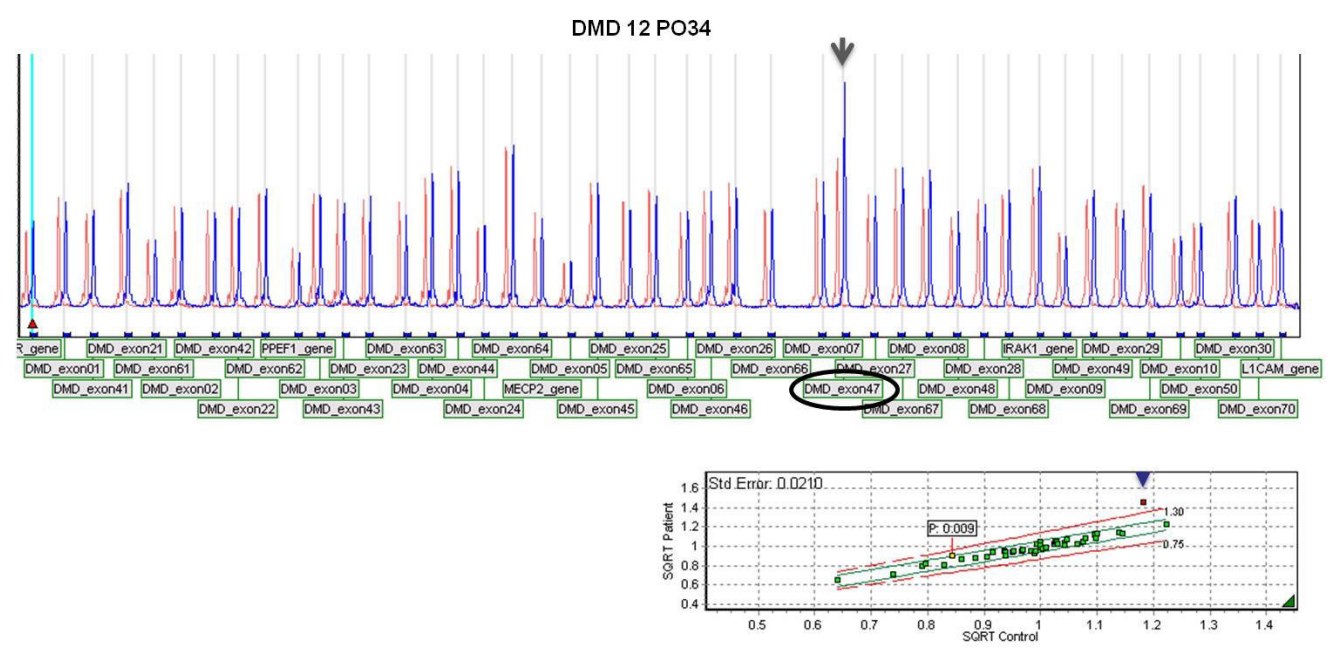

Figure 2. Multiplex ligation-dependent probe amplification (MLPA)-based duplication detection in an affected male without previous molecular diagnosis. Electropherogram analyzed by the Gene Marker version 1.6 of the MLPA assay on the affected male sample DMD 12 (blue peaks) compared to a healthy male control (red peaks). Probe mix PO34 revealed a duplication of exon 47 (black arrow), also detectable in the regression analysis shown in the box below. The red dot upon the green line corresponds to the duplicated exon. 
In the second male sample (DMD 11), MLPA analysis revealed a deletion of a single exon (number 38) (Figure 3A). The manufacturers recommend careful analysis of single exon

A

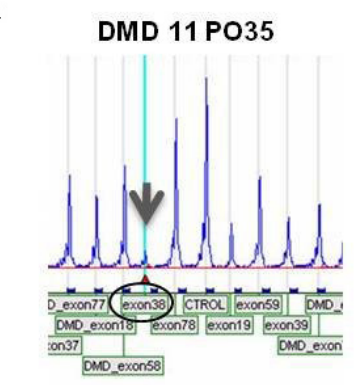

Healthy female control PO35

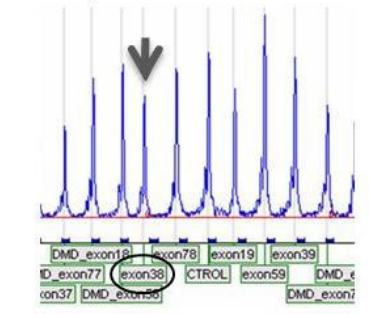

Healthy male control PO35

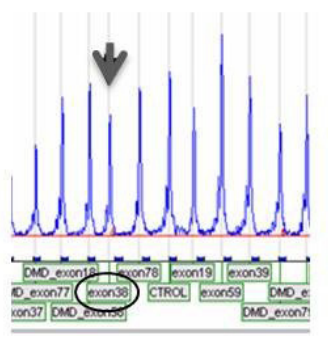

B

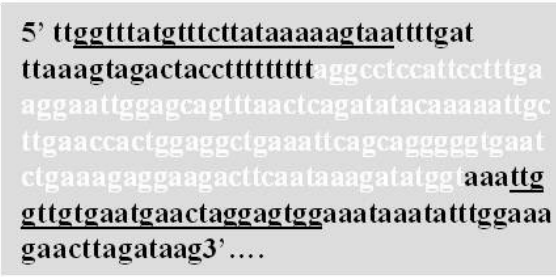

C
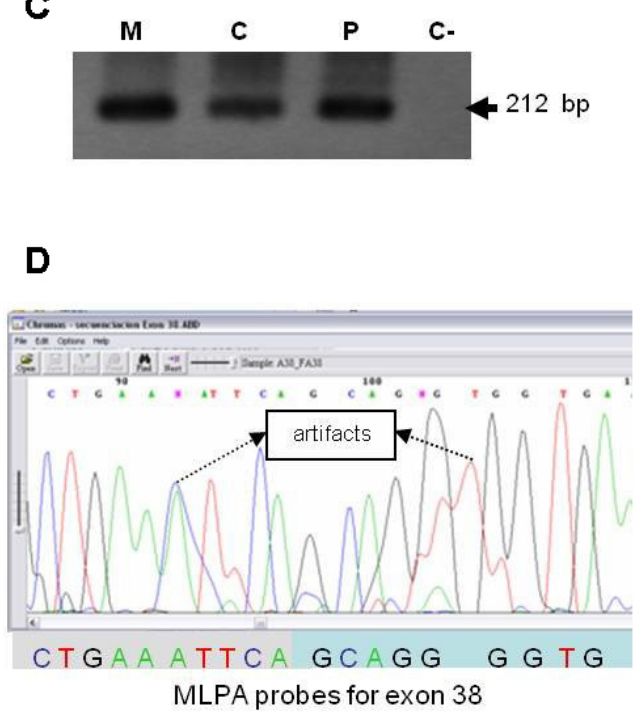

MLPA probes for exon 38

Figure 3. Multiplex ligation-dependent probe amplification (MLPA), polymerase chain reaction (PCR) and sequencing analysis of the male patient DMD 11. A. Electropherograms analyzed by the Gene Marker version 1.6 software of three samples: the first panel corresponds to the male patient DMD 11 analyzed with the probe mix PO35 (cover exons 11-20, 31-40, 51-60, and 71-79). The arrow indicates the absence of a peak at the position of exon 38. In the second and third panels, the electropherograms of a healthy female and male control are shown, which were analyzed with the same probe mix as used for patient DMD 11. Arrows show the presence of the peak at the position of exon 38 in both control samples. B. Two primers (underlined) were designed flanking exon 38 (white font) to produce a PCR product of $212 \mathrm{bp}$. C. PCR was performed on the mother DMD 7 (lane M), on a healthy female control (C) and on the male patient DMD 11 (lane P) and afterwards resolved on a 1.5\% agarose gel. Lane $C$ - shows a negative control of PCR without DNA sample. The 212-bp band revealed the presence of exon 38 in the patient and his mother. D. Electropherogram analyzed by the Sequencing Analysis version 5.2 software shows the sequence of the PCR product of exon 38 in the male patient DMD 11. It is worth noting that the male sample is hemizygous and can therefore only show one peak per nucleotide site. The black arrows indicate interfering noise-background that are considered artifacts of the reaction and that do not correspond to the real sequence of the PCR product. The gray- and light blue-colored boxes show the MLPA probe sequence for this exon. Notice the complete coincidence of the probe sequences with the electropherogram sequence. 
detection by MLPA. Any interfering component of the chemical reaction that could prevent the hybridization of one single probe would be observed in the final electropherograms as a lower peak (in case of a female sample) or as the complete absence of a peak (in case of a male sample), indeed corresponding to a false deletion. After obtaining the same MLPA profile twice in the affected boy, we analyzed his mother's DNA (DMD 7, Table 1B). This woman showed a completely healthy electropherogram, following which we could dismiss her carrier status. Therefore, in order to assess whether the boy had suffered a de novo mutation, or whether the absence of the peak could have resulted from a poor hybridization of a single probe, we developed a PCR-based strategy. Two primers were designed, flanking exon 38 and including the hybridization site of the MLPA probes for exon 38 (Figure 3B). PCR amplification was performed on a DNA sample from a healthy female control, in addition to the DNA from the carrier mother and from the affected male patient. The PCR products were resolved on an agarose gel, revealing the presence of a 212-bp band in all of the 3 samples (Figure 3C). These results suggest that there was no deletion at this site in the patient sample. As a second hypothesis, we wanted to determine whether any point mutation could have been affecting the hybridization site of any of the MLPA probes for exon 38. We analyzed the PCR product of the affected DMD 11 patient by direct sequencing. The results, however, did reveal a wild-type nucleotide sequence in the patient's sample (Figure 3D). This allowed us to discard the apparent deletion of exon 38 in this boy.

Of the remaining female carriers, one sample (DMD 5) showed a deletion in exons 48, 49 and 50 (Table 1B and Figure 4A). Her affected son had a previous PCR diagnosis of a deletion of exon 49. This previous result was consistent with the MLPA diagnosis of the carrier mother. Due to the severity of the illness, it was impossible to perform an MLPA analysis on this affected boy. In this case, the MLPA detection allowed the inference of the limits of the deletion of the son, and to predict his phenotype. The information about the complete deleted segment permitted the prediction of an out of frame deletion (Figure 4B). This is related to a DMD phenotype, rather than a milder Becker phenotype. In the last female sample (DMD 6), no deletion/duplication was detected. As she had a DMD-affected son who died without any PCR diagnosis, and in order to assess her carrier condition, direct sequencing of her complete DMD gene should be performed. This method is not available in Argentina and our group is contacting foreign diagnostic laboratories to send this single sample for analysis abroad.

\section{DISCUSSION}

The identification of female carriers is crucial for families with boys who have been diagnosed with the Duchenne-Becker illness. In Argentina, this diagnosis is performed by multiplex PCR, spanning 16 of the 79 exons. This leaves a high percentage (around 30\%) of genetically undiagnosed boys. In Argentina, the STR analysis is available for affected DMD families, although this is limited to a small percent of the entire coding region and not always used for routine diagnosis, because of the high complexity and time-consuming assay.

In this study, we investigated the applicability of MLPA for the identification of female carriers, and for the delimitation of the deleted fragments in males. To verify the sensitivity of the method, we first analyzed seven previously investigated samples with known deletions, which had been detected by STR analysis. Our results showed a 100\% confirmation of the deleted exons; moreover, in four of the seven cases, the MLPA assay was able to refine the breakpoints involved.

The subsequently performed diagnosis lacked previous investigations, and in two of 
A
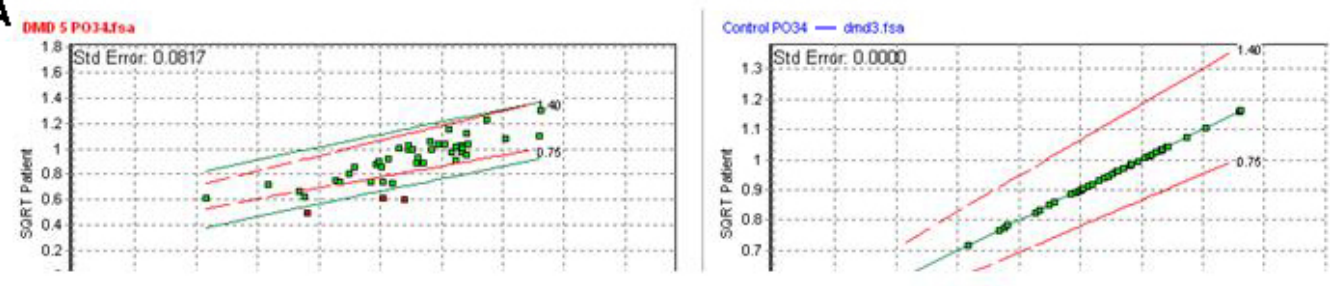

B

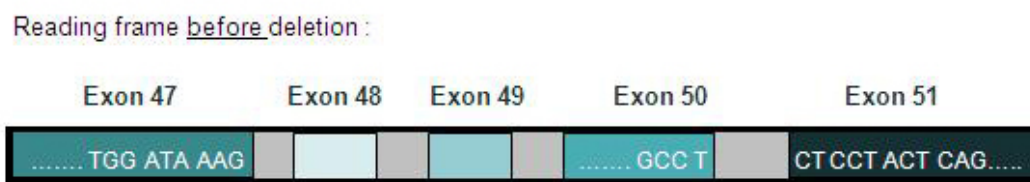

Reading frame after deletion

Exon $47 \quad$ Exon 51

\begin{tabular}{l|l} 
TGG ATA AAG & CTC CTA CTC AGA CTG TTA CTC TGG
\end{tabular}

Figure 4. Multiplex ligation-dependent probe amplification (MLPA)-based deletion detection in a female carrier without previous molecular diagnosis. A. MLPA regression analysis by the GeneMarker version 1.6 software is shown. This analysis relies upon a T-distribution to form the regression line between the square root of the sample and the square root of the control. The software forms a regression line until it has reached the desired confidence of $99.0 \%$. The left box corresponds to MLPA analysis on the female sample DMD 5 and the right box shows a healthy female control. The red dots below the green line in the left box correspond to the three deleted exons. B. Frame shift generated by the deletion is shown. Notice how the reading frame of exon 51 is changed after the deletion of exons 48, 49 and 50. The second and third codon positions of the first bases $\mathrm{C}$ and $\mathrm{T}$ are changed to first and second codon positions. This generates the complete shift of the reading frame, producing the spontaneous formation of the premature stop codon TGA, 21 nucleotides downstream.

five samples a deletion and a duplication were found by MLPA. Several exons were involved in the detected deletion. When more than one exon is deleted, the absence of many fragments serves as an internal control, given that the affected exons respect a consecutive numeric order. In one of the male samples, however, only a single exon appeared to be deleted, shown by MLPA. This should always be verified by an alternative method, because in such a case no other deleted exons can serve as control. We could discard this apparent result, checking the region by PCR and later sequencing. The explanation we propose is that one of the probes for exon 38 had poorly hybridized on the DNA sample of this particular sample, probably due to secondary structures of the DNA in that region.

The setting up and validation of this new method in Argentina will allow the development of more sensitive and specific diagnoses, and will provide useful information for the genetic counseling of affected Argentine families. This advance can be useful for other developing countries which still depend on foreign studies to perform local genetic diagnosis. The development and application of methods that improve genetic diagnosis will in the long term enhance prevention and clinical treatment strategies in countries such as Argentina. 


\section{ACKNOWLEDGMENTS}

We thank Dr. Gerard Pals and Dr. Jan Schouten (MRC-Holland) of the Vrije Universiteit, Amsterdam, The Netherlands, for the MLPA probes and for all their subsequent support. We thank Dr. A.L. Vargas, head of the Genetics Institute of the National University of Cuyo, Mendoza, for the financial support in the use of the automated sequencer. We also thank Katherine Joekes and Daniel Hayes for their meticulous revision of the manuscript.

\section{REFERENCES}

Abbs S and Bobrow M (1993). Report on the 16th ENMC workshop - carrier diagnosis of Duchenne and Becker muscular dystrophy. Neuromuscul. Disord. 3: 241-242.

Beggs AH, Koenig M, Boyce FM and Kunkel LM (1990). Detection of $98 \%$ of DMD/BMD gene deletions by polymerase chain reaction. Hum. Genet. 86: 45-48.

Bushby KM, Thambyayah M and Gardner-Medwin D (1991). Prevalence and incidence of Becker muscular dystrophy. Lancet 337: 1022-1024.

Chamberlain JS, Gibbs RA, Ranier JE, Nguyen PN, et al. (1988). Deletion screening of the Duchenne muscular dystrophy locus via multiplex DNA amplification. Nucleic Acids Res. 16: 11141-11156.

Clemens PR, Fenwick RG, Chamberlain JS, Gibbs RA, et al. (1991). Carrier detection and prenatal diagnosis in Duchenne and Becker muscular dystrophy families, using dinucleotide repeat polymorphisms. Am. J. Hum. Genet. 49: 951-960.

Darras BT, Harper JF and Francke U (1987). Prenatal diagnosis and detection of carriers with DNA probes in Duchenne's muscular dystrophy. N. Engl. J. Med. 316: 985-992.

Den Dunnen JT, Grootscholten PM, Bakker E, Blonden LA, et al. (1989). Topography of the Duchenne muscular dystrophy (DMD) gene: FIGE and cDNA analysis of 194 cases reveals 115 deletions and 13 duplications. Am. J. Hum. Genet. 45: 835-847.

Den Dunnen JT, Grootscholten PM, Dauwerse JG, Walker AP, et al. (1992). Reconstruction of the 2.4 Mb human DMDgene by homologous YAC recombination. Hum. Mol. Genet. 1: 19-28.

Ervasti JM and Campbell KP (1991). Membrane organization of the dystrophin-glycoprotein complex. Cell 66: 1121-1131.

Ervasti JM and Campbell KP (1993). A role for the dystrophin-glycoprotein complex as a transmembrane linker between laminin and actin. J. Cell Biol. 122: 809-823.

Ferreiro V (2006). Polimorfismo del ADN: su aplicacion en el diagnostico de enfermedades geneticas. (Polymorphisms in DNA: applications in the diagnosis of genetic diseases). PhD thesis, Universidad de Buenos Aires, Facultad de Farmacia y Bioquímica Catedra de Genetica y Biología Molecular, Buenos Aires.

Hoffman EP, Brown RH Jr and Kunkel LM (1987). Dystrophin: the protein product of the Duchenne muscular dystrophy locus. Cell 51: 919-928.

Koenig M, Hoffman EP, Bertelson CJ, Monaco AP, et al. (1987). Complete cloning of the Duchenne muscular dystrophy (DMD) cDNA and preliminary genomic organization of the DMD gene in normal and affected individuals. Cell 50: 509-517.

Ligon AH, Kashork CD, Richards CS and Shaffer LG (2000). Identification of female carriers for Duchenne and Becker muscular dystrophies using a FISH-based approach. Eur. J. Hum. Genet. 8: 293-298.

Petrof BJ (1998). The molecular basis of activity-induced muscle injury in Duchenne muscular dystrophy. Mol. Cell Biochem. 179: 111-123.

Prior TW, Papp AC, Snyder PJ, Highsmith WE Jr, et al. (1990). Determination of carrier status in Duchenne and Becker muscular dystrophies by quantitative polymerase chain reaction and allele-specific oligonucleotides. Clin. Chem. 36: 2113-2117.

Schouten JP, McElgunn CJ, Waaijer R, Zwijnenburg D, et al. (2002). Relative quantification of 40 nucleic acid sequences by multiplex ligation-dependent probe amplification. Nucleic Acids Res. 30: e57.

Sellner LN and Taylor GR (2004). MLPA and MAPH: new techniques for detection of gene deletions. Hum. Mutat. 23: 413-419.

Voskova-Goldman A, Peier A, Caskey CT, Richards CS, et al. (1997). DMD-specific FISH probes are diagnostically useful in the detection of female carriers of DMD gene deletions. Neurology 48: 1633-1638.

Yoshida M and Ozawa E (1990). Glycoprotein complex anchoring dystrophin to sarcolemma. J. Biochem. 108: 748-752. 